\title{
Odds Generalized Exponential-Inverse Weibull Distribution: Properties \& Estimation
}

\author{
Amal Soliman Hassan \\ Mathematical Statistics, Cairo University \\ Institute of Statistical Studies and Research, Giza, Egypt \\ dr.amalelmoslamy@gmail.com \\ Elsayed Ahmed Elsherpieny \\ Mathematical Statistics, Cairo University \\ Institute of Statistical Studies and Research, Giza, Egypt \\ ahmedc55@yahoo.com \\ Rokaya Elmorsy Mohamed \\ Mathematical Statistics, Cairo University \\ Institute of Statistical Studies and Research, Giza, Egypt \\ rokayaelmorsy@gmail.com
}

\begin{abstract}
Providing extended and generalized distribution is usually precious for many statisticians. A new distribution, called odds generalized exponential-inverse Weibull distribution (OGE-IW) is suggested for modeling lifetime data. Some structural properties of the new distribution are obtained. Three different estimation procedures, namely; maximum likelihood, percentiles and least squares, are used to estimate the model parameters of subject distribution. The consistency of the parameters of the OGE-IW distribution is demonstrated through a simulation study. A real data application is presented to illustrate the importance of the new distribution compared with some known distributions.
\end{abstract}

Keywords: T-X family, Inverse Weibull distribution; Maximum likelihood estimators; Least squares estimators, Percentiles estimators.

\section{Introduction}

The inverse Weibull (IW) distribution has been received some attention in the literature. The IW distribution can be used to a diverse model of failure characteristics, such as infant mortality, age of production, and periods of erosion. The inverse Weibull distribution can also be used to determine the cost-effectiveness and maintenance periods of reliability centered maintenance activities. An early study about IW model has been developed by Erto in 1989. The shapes of the density and failure rate functions for the basic inverse model have been studied by Keller and Kamath (1982). The maximum likelihood and least squares estimators of IW distribution have been studied by Calabria and Pulcini (1990). Bayes two-sample prediction of the IW distribution has been developed by Calabria and Pulcini (1994). Hassan and AL-Thobety (2012) provided an optimal design of failure step stress partially accelerated life tests with type II inverse Weibull data. Hassan et al. (2015) studied constant stress partially accelerated life tests with Type II inverse Weibull data using multiple censored data. The probability density 
function (pdf) and cumulative distribution function (cdf) of IW distribution with shape parameter $\alpha$ and scale parameter $\beta$ are given, respectively, by

$$
\begin{aligned}
& g(x ; \beta, \alpha)=\alpha \beta x^{-\alpha-1} e^{-\beta x^{-\alpha}} ; \quad \alpha, \beta>0, \quad x>0, \\
& G(x ; \beta, \alpha)=e^{-\beta x^{-\alpha}} .
\end{aligned}
$$

Extended and generalized forms of IW distribution are studied by some authors, among them; Khan (2010) introduced and studied the beta inverse Weibull distribution. de Gusmão et al. (2011) introduced three-parameter inverse Weibull distribution, called the generalized inverse Weibull distribution, with unimodal, increasing and decreasing failure rates. Khan and King (2012) proposed four-parameter modified inverse Weibull distribution. Shahbaz et al. (2012) suggested the Kumaraswamy inverse Weibull distribution. Elbatal and Muhammed (2014) introduced the exponentiated generalized inverse Weibull distribution. The generalized inverse Weibull distribution including the exponentiated or proportional reverse hazard and Kumaraswamy generalized inverse Weibull distributions have been suggested by Oluyede and Yang (2014). Pararai et al. (2014) introduced gamma-inverse Weibull distribution based on gamma generated family. Khan et al. (2014) studied characterizations of the transmuted inverse Weibull distribution with an application to bladder cancer remission time's data. Khan and King (2016) introduced the four-parameter new generalized inverse Weibull distribution and investigated its potential usefulness with application to reliability data from engineering studies. Rodrigues et al. (2016) introduced exponentiated Kumaraswamy inverse Weibull distribution. Okasha et al. (2017) introduced the Marshall-Olkin extended inverse Weibull distribution.

The statistics literature is filled with lots of continuous univariate distributions for describing real data. In recent years, there has been a great interest among statisticians and applied researchers in constructing flexible distribution to facilitate better modeling of lifetime data in various situations. Several methods have been developed for generating new family of lifetime distributions. One approach of generalization was suggested by Marshall and Olkin (1997) by adding one parameter to the survival function $\bar{G}(x)$. In the same trend, Gupta et al. (1998) added one parameter to the cdf $G(x)$ of the baseline distribution to define the exponentiated $-\mathrm{G}$ class of distribution. Following Gupta's et al. class, Gupta and Kundu (1999) studied the two-parameter generalized exponential distribution as an extension of the exponential distribution. Our interest here with T-X family proposed by Alzaatreh et al. (2013), the cdf of T-X family is specified by

$$
F(x)=\int_{0}^{W(G(x)} f(t) d t,
$$

where, the random variable $T$ called the transformer and $W(G(x))$ be a function of $G(x)$. Based on T-X family; Tahir et al. (2015) introduced the odd generalized exponential by using the generalized exponential as generator in (3) and taking the upper limit to be $G(x) / \bar{G}(x)$, the odds function of any distribution. Maiti and Pramanik (2015) defined a generalized class of any distribution by taking the exponential distribution as a generator in (3) and taking the upper limit to be $G(x) / \bar{G}(x)$. Further, 
Alizadeh et al. (2017) proposed and studied a new generated family called the generalized odd generalized exponential.

Our motivation here is to introduce and study a new extended form for the inverse Weibull distribution with three parameters. We call the new distribution; the odds generalized exponential-inverse Weibull distribution, which is a particular case of T-X family of distributions. The rest of the paper contains the following sections. The new distribution is provided in Section 2. Some statistical properties are given in Section 3. Then, in Section 4, maximum likelihood, least squares and percentiles estimators are obtained. Simulation study and results are presented in Section 5. An application of the OGE-IW model to real data is presented in Section 6. At the end, concluding remarks are addressed in Section 7.

\section{Construction of the OGE-IW Distribution}

In this section, the pdf, cdf, reliabilty function, hazard rate function (hrf), reversed-hazard rate function and cumulative hazard rate function of OGE-IW distribution are derived. Expansions for its pdf and cdf are also provided.

We obtain the OGE-IW distribution by considering the exponential distribution as transformer in cdf (3); also, taking; $W(G(x))=G(x) / \bar{G}(x)$, the odds ratio of inverse Weibull distribution defined in (2) as follows

$$
F(x ; \alpha, \lambda, \beta)=\int_{0}^{\frac{G(x)}{1-G(x)}} \lambda e^{-\lambda t} d t=\int_{0}^{\frac{e^{-\beta x^{-\alpha}}}{1-e^{-\beta x} x^{-\alpha}}} \lambda e^{-\lambda t} d t
$$

Hence, the cdf of OGE-IW distribution is as follows

$$
F(x ; \alpha, \lambda, \beta)=1-\exp -\left(\frac{\lambda}{e^{\beta x^{-\alpha}}-1}\right), \quad x>0
$$

The corresponding pdf is obtained as follows

$$
f(x ; \alpha, \lambda, \beta)=\alpha \beta \lambda x^{-\alpha-1} e^{-\beta x^{-\alpha}}\left(1-e^{-\beta x^{-\alpha}}\right)^{-2} \exp -\left(\frac{\lambda}{e^{\beta x^{-\alpha}}-1}\right) .
$$

For $\alpha=2$, the OGE-IW reduces to a new model named as odds generalized exponential inverse Rayleigh distribuiton. For $\alpha=1$, OGE-IW reduces to another new model named as odds generalized exponential inverse exponential distribuiton.

Plots of the pdf of OGE-IW distribution for some selected parameter values are displayed in Figure 1. As seems from this figure, the pdf of OGE-IW distribution can be symmetric, unimodel and right skewed according to the selected values of parameters. 


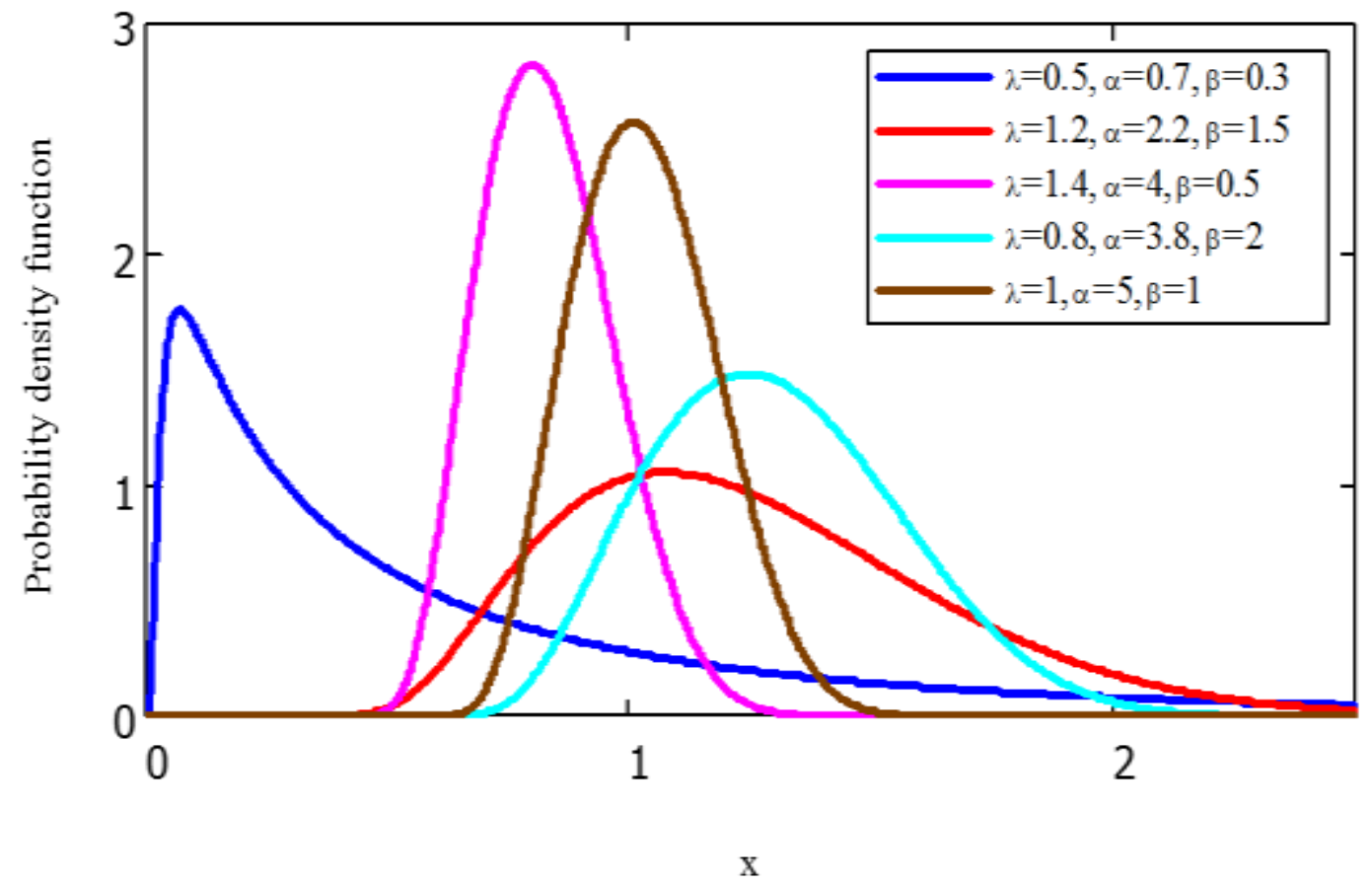

Figure 1: Plots of the pdf of OGE-IW distribution for selected values of the parameters

\subsection{Expansion for Densities of OGE-IW Distribution}

Two useful expansions of OGE-IW pdf and cdf are derived. Since, the pdf (5) can be rewritten as follows

$$
f(x ; \lambda, \alpha, \beta)=\alpha \beta \lambda x^{-\alpha-1} e^{-\beta x^{-\alpha}}\left(1-e^{-\beta x^{-\alpha}}\right)^{-2} \exp -\lambda\left(\frac{e^{-\beta x^{-\alpha}}}{1-e^{-\beta x^{-\alpha}}}\right) .
$$

Then, by using the exponential expansion for the last term in (6) and further the binomial expansion for a positive real power yields

$$
f(x ; \lambda, \alpha, \beta)=\sum_{j, i=0}^{\infty}(-1)^{j} \frac{\alpha \beta \lambda^{j+1}}{j !} \frac{\Gamma(j+2+i)}{\Gamma(j+2) i !} x^{-\alpha-1} e^{-\beta(j+i+1) x^{-\alpha}} .
$$

Then the pdf (7) can be formed as follows

$$
f(x ; \lambda, \alpha, \beta)=\sum_{j, i=0}^{\infty} c_{j, i} g_{\beta(j+i+1)}(x),
$$

where, denotes the pdf of IW distribution $g_{\beta(j+i+1)}(x)$ and $c_{j, i}=(-1)^{j} \frac{\lambda^{j+1}}{j !} \frac{\Gamma(j+i+1)}{\Gamma(j+2) i !}$ with parameters $\beta(j+i+1)$ and $\alpha$. 
Further, an expansion for $[F(x ; \lambda, \alpha, \beta)]^{t}$, for $t$ a positive real power is derived as follows

$$
[F(x ; \lambda, \alpha, \beta)]^{t}=\sum_{m=0}^{t} \sum_{l, p=0}^{\infty} \omega_{m, l, p} G_{\beta(l+p)}(x),
$$

where, $\omega_{m, l, p}=(-1)^{m+l}\left(\begin{array}{c}t \\ m\end{array}\right) \frac{(\lambda m)^{l} \Gamma(l+p)}{p ! l ! \Gamma(l)}$ and $G_{\beta(l+p)}(x)$ is the cdf of IW with parameters $\beta(l+p)$ and $\alpha$.

\subsection{Reliability Analysis}

This subsection gives expressions for the reliability function, hazard function, and reversed hazard function.

The survival function and hrf of the OGE-IW distribution are respectively given by

$$
\begin{aligned}
& \bar{F}(x ; \alpha, \lambda, \beta)=\exp -\left(\frac{\lambda}{e^{\beta x^{-\alpha}}-1}\right), \\
& h(x ; \alpha, \lambda, \beta)=\lambda \alpha \beta x^{-\alpha-1}\left(1-e^{-\beta x^{-\alpha}}\right)^{-2} .
\end{aligned}
$$

Figure 2 gives the plots of the hrf of OGE-IW distribution for some selected parameter values. Figure 2 indicates that OGE-IW hrfs can have increasing, decreasing and constant. This fact implies that the OGE-IW can be very useful for fitting data sets with various shapes.

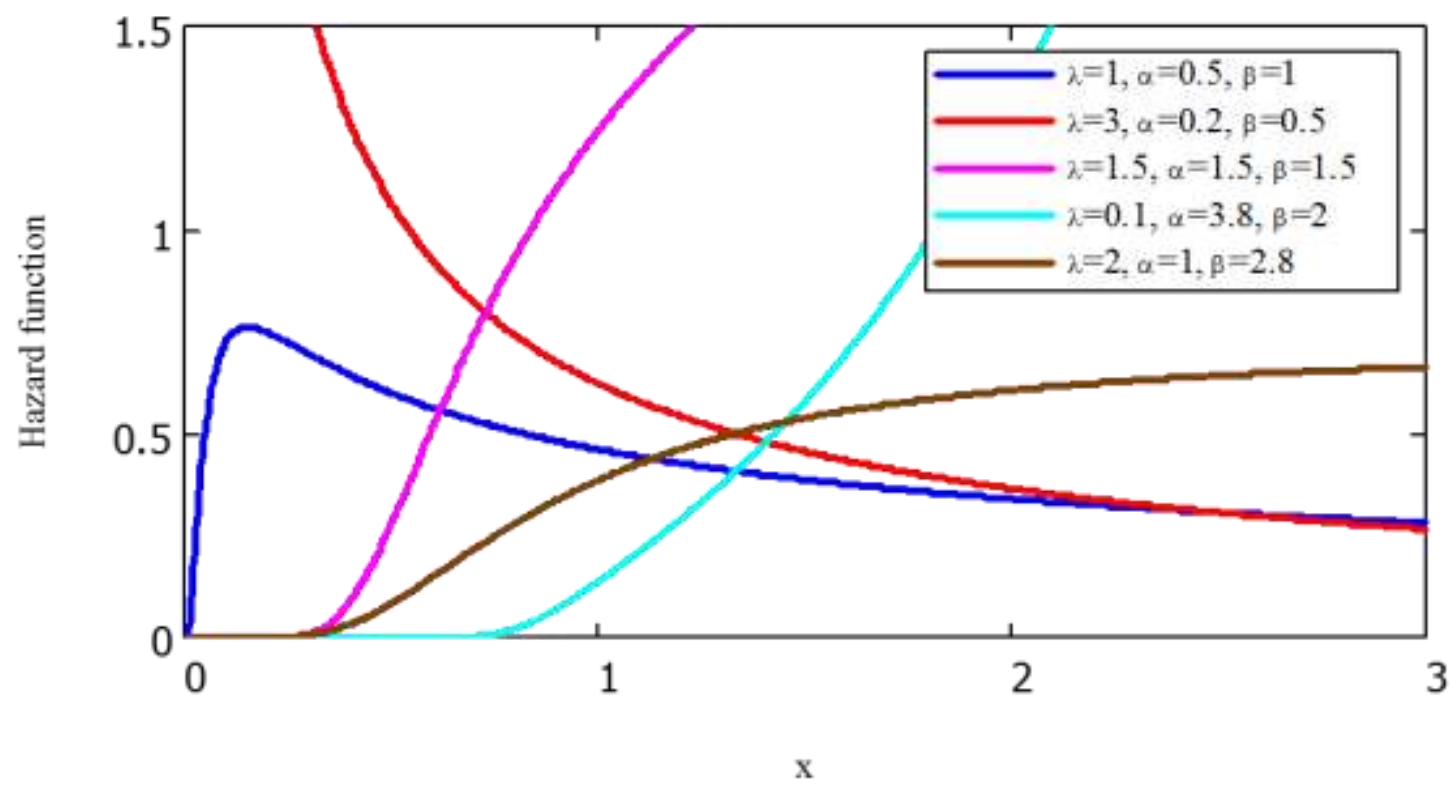

Figure 2: Plots of hrf of OGE-IW distribution for selected values of the parameters 
The reversed-hazard rate function of the OGE-IW distribution is as follows

$$
v(x ; \alpha, \lambda, \beta)=\frac{\lambda \alpha \beta x^{-\alpha-1} e^{-\beta x^{-\alpha}}}{\left(1-e^{-\beta x^{-\alpha}}\right)^{2}\left[\exp -\left(\frac{\lambda}{e^{\beta x^{-\alpha}}-1}\right)-1\right]},
$$

Additionaly, the cumulative hazard rate function of the OGE-IW is given by

$$
H(x ; \alpha, \lambda, \beta)=-\ln \left|\exp -\left(\frac{\lambda}{e^{\beta x^{-\alpha}}-1}\right)\right| \text {. }
$$

\section{Some Mathematical Properties}

In this section, some mathematical properties of the OGE-IW distribution, including, moments, probability weighted moments, incomplete moments, order statistics and entropy measure are derived.

\subsection{Moments}

The rth moment of OGE-IW is derived by using pdf (8) as follows

$$
\mu_{r}^{\prime}=\sum_{j, i=0}^{\infty} c_{j, i} \int_{0}^{\infty} x^{r} g_{\beta(j+i+1)}(x) d x=\sum_{j, i=0}^{\infty} c_{j, i}[\beta(j+i+1)]^{\frac{r}{\alpha}} \Gamma\left(1-\frac{r}{\alpha}\right), r=1,2,3, \ldots
$$

In particular, the mean and variance of the OGE-IW distribution are given by

$$
\begin{aligned}
& E(X)=\sum_{j, i=0}^{\infty} c_{j, i}[\beta(j+i+1)]^{\frac{1}{\alpha}} \Gamma\left(1-\frac{1}{\alpha}\right), \\
& \operatorname{var}(X)=\sum_{j, i=0}^{\infty} c_{j, i}[\beta(j+i+1)]^{\frac{1}{\alpha}} \Gamma\left(1-\frac{2}{\alpha}\right)-\left[\sum_{j, i=0}^{\infty} c_{j, i}[\beta(j+i+1)]^{\frac{1}{\alpha}} \Gamma\left(1-\frac{1}{\alpha}\right)\right]^{2}
\end{aligned}
$$

The skewness $\left(g_{1}\right)$ of the OGE-IW distribution is given by

$$
g_{1}=\frac{\mu_{3}^{2}}{\mu_{2}^{3}}=\frac{\left(\mu_{3}^{\prime}-3 \mu_{2}^{\prime} \mu_{1}^{\prime}+2 \mu_{1}^{\prime 3}\right)^{2}}{\left(\mu_{2}^{\prime}-\mu_{1}^{\prime 2}\right)^{3}} \text {. }
$$

The kurtosis $\left(g_{2}\right)$ of the OGE-IW is given by

$$
g_{2}=\frac{\mu_{4}}{\mu_{2}^{2}}=\frac{\mu_{4}^{\prime}-4 \mu_{3}^{\prime} \mu_{1}^{\prime}+6 \mu_{2}^{\prime} \mu_{1}^{\prime 2}-3 \mu_{1}^{\prime 4}}{\left(\mu_{2}^{\prime}-\mu_{1}^{2}\right)^{2}} \text {. }
$$

Table (1) provides the mean $(\mu)$ and variance (var) of the OGE-IW distribution for various parameter values. From Table (1), we notice that both values of the mean and variance of the OGE-IW decrease as the values of $\alpha$ and $\lambda$ increases. Also, the values of the mean and variance increase as the values of $\beta$ increase. Table (2) contains the skewness and kurtosis of the OGE-IW distribution for various values of parameters $\alpha$ and $\lambda$.We notice that both the skewness and the kurtosis are decreasing functions of $\alpha$ and $\lambda$. 
Table 1: Mean and variance of OGE-IW distribution for various values of $\alpha, \lambda$ and $\beta$

\begin{tabular}{|c|c|cc|cc|cc|}
\hline \multicolumn{2}{|c|}{} & \multicolumn{2}{c|}{$\beta=0.5$} & \multicolumn{2}{c|}{$\beta=1$} & \multicolumn{2}{c|}{$\beta=2.5$} \\
\hline$\alpha$ & $\lambda$ & $\mu$ & var & $\mu$ & var & $\mu$ & var \\
\hline \multirow{3}{*}{2} & 1 & 0.7900 & 0.0800 & 1.1180 & 0.1600 & 1.7670 & 0.4000 \\
\cline { 2 - 8 } & 2 & 0.6330 & 0.0370 & 0.8960 & 0.0470 & 1.4160 & 0.1840 \\
\cline { 2 - 8 } & 3 & 0.5660 & 0.0240 & 0.8010 & 0.0470 & 1.2660 & 0.1180 \\
\hline \multirow{3}{*}{4.5} & 1 & 0.8870 & 0.0200 & 1.0350 & 0.0270 & 1.2680 & 0.0400 \\
\cline { 2 - 8 } & 2 & 0.8070 & 0.0120 & 0.9420 & 0.0160 & 1.1540 & 0.0240 \\
\cline { 2 - 8 } & 3 & 0.7700 & 0.0085 & 0.8980 & 0.0740 & 1.1010 & 0.0170 \\
\hline \multirow{3}{*}{5} & 1 & 0.8970 & 0.0160 & 1.0300 & 0.0220 & 1.2370 & 0.0310 \\
\cline { 2 - 8 } & 2 & 0.8240 & 0.0098 & 0.9470 & 0.0130 & 1.1370 & 0.0190 \\
\cline { 2 - 8 } & 3 & 0.7900 & 0.0072 & 0.9070 & 0.0096 & 1.0890 & 0.0140 \\
\hline
\end{tabular}

Table 2: Skewness and kurtosis of OGE-IW distribution for various values of $\alpha$ and $\lambda$

\begin{tabular}{|c|c|cc|}
\hline$\alpha$ & $\lambda$ & $g_{1}$ & $g_{2}$ \\
\hline \multirow{3}{*}{2} & 1 & 0.7910 & 3.6220 \\
\cline { 2 - 4 } & 2 & 0.7530 & 3.6210 \\
\cline { 2 - 4 } & 3 & 0.7040 & 3.5700 \\
\hline \multirow{4}{*}{4.5} & 1 & 0.2940 & 2.7570 \\
\cline { 2 - 4 } & 2 & 0.3100 & 2.8560 \\
\cline { 2 - 4 } & 3 & 0.2950 & 2.8980 \\
\hline \multirow{3}{*}{5} & 1 & 0.2550 & 2.7230 \\
\cline { 2 - 4 } & 2 & 0.2750 & 2.8240 \\
\cline { 2 - 4 } & 3 & 0.2630 & 2.8690 \\
\hline
\end{tabular}

Furthermore, the moment generating function of OGE-IW can be obtained as follows

$$
M_{x}(t)=\sum_{r=0}^{\infty} \frac{t^{r}}{r !} E\left(X^{r}\right)=\sum_{r=0}^{\infty} \sum_{j, i=0}^{\infty} c_{j, i} \frac{t^{r}[\beta(j+i+1)]^{\frac{r}{\alpha}} \Gamma\left(1-\frac{r}{\alpha}\right)}{r !}, r=1,2, \ldots
$$

\subsection{Probability Weighted Moments (PWMs)}

Greenwood et al. (1979) introduced the probability weighted moments to derive estimators of the parameters and quantiles of distributions. The PWMs of OGE-IW distribution is defined by

$$
\tau_{r, t}=E\left\{X^{r}[F(x)]^{t}\right\}=\int_{-\infty}^{\infty} x^{r}[F(x)]^{t} f(x) d x,
$$


where, $t$ and $r$ are positive integers. Inserting pdf (8) and cdf (9) in (11), then the PWMs of the OGE-IW distribution is obtained as follows

$$
\tau_{r, t}=\sum_{m=0}^{t} \sum_{l, p, j, i=0}^{\infty} \omega_{m, l, p} c_{j, i} \frac{\beta(j+i+1) \Gamma\left(1-\frac{r}{\alpha}\right)}{[\beta(j+i+p+l+1)]^{1-\frac{r}{\alpha}}} .
$$

\subsection{Incomplete Moments}

Theis defined by $£_{s}(a)$, moment, sayincomplete sth

$$
£_{s}(a)=\int_{-\infty}^{a} x^{s} f(x) d x
$$

Hence, the sth moment of OGE-IW is derived by inserting (8) in (12) as follows

$$
£_{s}(a)=\sum_{j, i=0}^{\infty} c_{j, i} \beta(j+i+1)^{\frac{s}{\alpha}} \Gamma\left(1-\frac{s}{\alpha}, \beta(j+i+1) a^{-\alpha}\right) .
$$

where, $\Gamma\left(1-\frac{s}{\alpha}, \beta(j+i+1) a^{-\alpha}\right)$ is the upper incomplete gamma function. In particular, the first incomplete moments of the OGE-IW distribution can be obtained by putting $s=1$ in (13), as follows

$$
£_{1}(a)=\sum_{j, i=0}^{\infty} c_{j, i} \beta(j+i+1)^{\frac{1}{\alpha}} \Gamma\left(1-\frac{1}{\alpha}, \beta(j+i+1) a^{-\alpha}\right) .
$$

Bonferroni and Lorenz curves are useful applications to first incomplete moments. These curves are very useful in economics, reliability, demography, insurance and medicine. The Lorenz and Bonferroni curves are obtained, respectively, as follows

$$
L_{F}(x)=\frac{1}{E(X)} \int_{0}^{x} a f(a) d a=\frac{\sum_{j, i=0}^{\infty} c_{j, i} \beta(j+i+1)^{\frac{1}{\alpha}} \Gamma\left(1-\frac{1}{\alpha}, \beta(j+i+1) x^{-\alpha}\right)}{\sum_{j, i=0}^{\infty} c_{j, i}[\beta(j+i+1)]^{\frac{1}{\alpha}} \Gamma\left(1-\frac{1}{\alpha}\right)},
$$

and

$$
B_{F}(x)=\frac{L_{F}(x)}{F(x)}=\frac{\sum_{j, i=0}^{\infty} c_{j, i} \beta(j+i+1)^{\frac{1}{\alpha}} \Gamma\left(1-\frac{1}{\alpha}, \beta(j+i+1) x^{-\alpha}\right)}{\left[1-\exp -\left(\frac{\lambda}{e^{\beta x^{-\alpha}}-1}\right)\right] \sum_{j, i=0}^{\infty} c_{j, i}[\beta(j+i+1)]^{\frac{1}{\alpha}} \Gamma\left(1-\frac{1}{\alpha}\right)} .
$$

Another application of the first incomplete moments refers to the mean deviations which provide useful information about the characteristics of a population. Indeed, the amount of dispersion in a population may be measured to some extent by the totality of the deviations from the mean and median. The mean deviations of $X$ about the meanand $\mu$ about the median $m$ can be calculated from the following relations

$$
\delta_{1}=2 \mu F(\mu)-2 T(\mu) \quad \text { and } \quad \delta_{2}=\mu-2 T(m),
$$


where, $T(q)=\int_{0}^{q} x f(x) d x$ which is the first incomplete moment, then from (14) $T(\mu)$ and $T(m)$ are obtained, respectively, as follows

$$
\begin{aligned}
& T(\mu)=\int_{0}^{\mu} x f(x) d x=\sum_{j, i=0}^{\infty} c_{j, i} \beta(j+i+1)^{\frac{1}{\alpha}} \Gamma\left(1-\frac{1}{\alpha}, \beta(j+i+1) \mu^{-\alpha}\right), \\
& T(m)=\int_{0}^{m} x f(x) d x=\sum_{j, i=0}^{\infty} c_{j, i} \beta(j+i+1)^{\frac{1}{\alpha}} \Gamma\left(1-\frac{1}{\alpha}, \beta(j+i+1) m^{-\alpha}\right) .
\end{aligned}
$$

\subsection{Rényi Entropy}

The entropy of a random variable $X$ with density function $f(x)$ is a measure of the uncertainty variation. The Rényi entropy is defined as

$$
I_{R}(\delta)=\frac{1}{1-\delta} \ln \left\{\int_{-\infty}^{\infty} f^{\delta}(x) d x\right\}
$$

where $\delta>0$ and $\delta \neq 1$. Applying the exponential and binomial expansions, then $f^{\delta}(x ; \lambda, \alpha, \beta)$ can be expressed as follows

$$
f^{\delta}(x ; \lambda, \alpha, \beta)=\sum_{j=0}^{\infty}(-1)^{j} \frac{(\alpha \beta)^{\delta} \delta^{j} \lambda^{j+\delta} x^{-\delta(\alpha+1)} \Gamma(j+i+2 \delta) e^{-\beta(j+\delta+i) x^{-\alpha}}}{j ! \Gamma(j+2 \delta) i !}\left(1-e^{-\beta x^{-\alpha}}\right)^{-(j+2 \delta)}
$$

Inserting (16) in (15), then the Rényi entropy of OGE-IW distribution becomes

$$
I_{R}(\delta)=\frac{1}{1-\delta} \ln \left\{\sum_{j, i=0}^{\infty}(-1)^{j} \frac{(\beta)^{\delta} \alpha^{\delta-1} \delta^{j} \lambda^{j+\delta} \Gamma(j+2 \delta+i)}{j ! i ! \Gamma(j+2 \delta)[\beta(j+\delta+i)]^{\frac{\delta(\alpha+1)-1}{\alpha}}} \Gamma\left(\frac{\delta(\alpha+1)-1}{\alpha}\right)\right\}
$$

\subsection{Order Statistics}

Let $X_{1: n}<X_{2: n}<\ldots<X_{n: n}$ denote the order statistics for a random sample $X_{1}, X_{1}, \ldots, X_{n}$ from OGE-IW distribution with pdf (8) and cdf (9). The pdf of rth order statistics is defined by

$$
f_{r: n}(x)=\frac{1}{B(r, n-r+1)} \sum_{k=0}^{n-r}\left(\begin{array}{c}
n-r \\
k
\end{array}\right)(-1)^{k}[F(x)]^{k+r-1} f(x) .
$$

Using the binomial expansion for $[F(x)]^{k+r-1}$, replacing $t$ in (9) with $k+r-1$. Hence the pdf (17) becomes

$$
f_{r: n}(x)=\frac{1}{B(r, n-r+1)} \sum_{k=0}^{n-r} \sum_{m=0}^{k+r-1} \sum_{j, i, l, p=0}^{\infty} \eta_{k, j, i, m, l, p} x^{-\alpha-1} e^{-\beta(j+i+l+p+1) x^{-\alpha}},
$$

where

$$
\eta_{k, j, i, m, l, p}=(-1)^{k+j+m+l}\left(\begin{array}{c}
n-r \\
k
\end{array}\right)\left(\begin{array}{c}
k+r-1 \\
m
\end{array}\right) \frac{\alpha \beta \lambda^{j+1} \Gamma(j+2+i)(\lambda m)^{l} \Gamma(p+l)}{\Gamma(j+2) \Gamma(l) j ! i ! l ! p !} .
$$


In particular, the pdf of the smallest order statistics is obtained by substituting $r=1$ in (18) as follows

$$
f_{1: n}(x)=n \sum_{k=0}^{n-1} \sum_{m=0}^{k+r-1} \sum_{j, i, l, p=0}^{\infty} \pi_{k, j, i, m, l, p} x^{-\alpha-1} e^{-\beta(j+i+l+p+1) x^{-\alpha}}
$$

where

$$
\pi_{k, j, i, m, l, p}=(-1)^{j+m+l}\left(\begin{array}{c}
n-1 \\
k
\end{array}\right)\left(\begin{array}{c}
k \\
m
\end{array}\right) \alpha \beta \lambda^{j+1} \frac{\Gamma(j+2+i)(\lambda m)^{l} \Gamma(p+l)}{\Gamma(j+2) \Gamma(l) j ! i ! l ! p !} .
$$

Also, the pdf of largest order statistics is obtained by substituting $r=n$ in (18) as follows

$$
f_{n: n}(x)=n \sum_{m=0}^{k+r-1} \sum_{j, i, l, p=0}^{\infty} v_{k, j, i, m, l, p} x^{-\alpha-1} e^{-\beta(j+i+l+p+1) x^{-\alpha}},
$$

where

$$
v_{k, j, i, m, l, p}=(-1)^{j+m+l}\left(\begin{array}{c}
k+n-1 \\
m
\end{array}\right) \alpha \beta \lambda^{j+1} \frac{\Gamma(j+2+i)(\lambda m)^{l} \Gamma(p+l)}{\Gamma(j+2) \Gamma(l) j ! i ! l ! p !} .
$$

\section{Parameter Estimation}

In this section, the parameter estimators of the OGE-IW model parameters are obtained based on maximum likelihood (ML), least squares (LS) and percentiles methods.

\subsection{Maximum Likelihood Estimators}

In this subsection, the estimation of the unknown parameters of the OGE-IW distribution is considered using the ML method. Let $X_{1}, \ldots, X_{n}$ be observed values from the OGE-IW distribution. The total $\log$-likelihood function, denoted by $\ln L$, for the parameters $\lambda, \alpha$ and $\beta$ in complete sample is as follows

$$
\ln L=\mathrm{n} \ln \alpha+\mathrm{n} \ln \beta+\mathrm{n} \ln \lambda-(\alpha+1) \sum_{i=1}^{n} \ln x_{i}-\beta \sum_{i=1}^{n} x_{i}{ }^{-\alpha}-\sum_{i=1}^{n}\left(\frac{\lambda}{e^{\beta x_{i}^{-\alpha}}-1}\right)-2 \sum_{i=1}^{n} \ln \left(1-e^{-\beta x_{i}{ }^{-\alpha}}\right) \text {. }
$$

The partial derivatives of the log-likelihood function with respect to $\lambda, \alpha$ and $\beta$ components of the score vector $U_{L}=\left(U_{\lambda}, U_{\alpha}, U_{\beta}\right)^{T}$ can be obtained as follows

$$
\begin{aligned}
& U_{\lambda}=\frac{\partial \ln L}{\partial \lambda}=\frac{n}{\lambda}-\sum_{i=1}^{n}\left(\frac{1}{e^{\beta x_{i}{ }^{-\alpha}}-1}\right) \\
& U_{\alpha}=\frac{\partial \ln L}{\partial \alpha}=\frac{n}{\alpha}-\sum_{i=1}^{n} \ln x_{i}+\beta \sum_{i=1}^{n} x_{i}^{-\alpha} \ln x_{i}-\sum_{i=1}^{n} \frac{\lambda \beta x_{i}^{-\alpha} \ln x_{i} e^{\beta x_{i}^{-\alpha}}}{\left(e^{\beta x_{i}^{-\alpha}}-1\right)^{2}}+2 \sum_{i=1}^{n} \frac{\beta x_{i}^{-\alpha} \ln x_{i} e^{-\beta x_{i}-\alpha}}{\left(1-e^{-\beta x_{i}-\alpha}\right)}, \\
& U_{\beta}=\frac{\partial \ln L}{\partial \beta}=\frac{n}{\beta}-\sum_{i=1}^{n} x_{i}^{-\alpha}+\sum_{i=1}^{n} \frac{\lambda x_{i}^{-\alpha} e^{\beta x_{i}}}{\left(e^{\beta x_{i}^{-\alpha}}-1\right)^{2}}-2 \sum_{i=1}^{n} \frac{x_{i}^{-\alpha}}{\left(e^{\beta x_{i}^{-\alpha}}-1\right)} .
\end{aligned}
$$


Then the maximum likelihood estimates (MLEs) of the parameters, denoted by $\hat{\lambda}, \hat{\alpha}$ and $\hat{\beta}$ are obtained by setting $U_{\lambda}, U_{\alpha}$ and $U_{\beta}$ to be zero and solving them numerically.

\subsection{Least Squares Estimator}

Suppose that $X_{1}, X_{2}, \ldots, X_{n}$ is a random sample of size $n$ from the OGE-IW distribution and suppose $X_{1: n}<X_{2: n}<\ldots<X_{n: n}$ denotes the corresponding ordered sample. The LS estimators of the unknown parameters $\lambda, \alpha$ and $\beta$ denoted by $\not / \%$ and $\beta^{k}$ of the OGEIW distribution can be obtained by minimizing the sum of squares errors with respect to $\lambda, \alpha$ and $\beta$,

$$
\sum_{i=1}^{n}\left[F_{i: n}(x)-\frac{i}{n+1}\right]^{2} .
$$

So the LS estimators $\not / \%$ land $\beta$ rof the OGE-IW model can be obtained by minimizing the following quantity

$$
\sum_{i=1}^{n}\left[\left[1-\exp -\left(\frac{\lambda}{e^{\beta x_{i}^{-\alpha}}-1}\right)\right]-\frac{i}{n+1}\right]^{2},
$$

with respect to $\lambda, \alpha$ and $\beta$ respectively.

\subsection{Percentiles Estimator}

Let $X_{1}, X_{2}, \ldots, X_{n}$ be a random sample from the OGE-IW, let $X_{i: n}$ denotes the $i t h$ order statistic, i.e, $X_{1: n}<X_{2: n}<\ldots<X_{n: n}$. If $p_{i}$ denotes some estimates of $F\left(x_{i: n} ; \lambda, \alpha, \beta\right)$, then the estimator of unknown parameters, denoted by $\bar{\lambda}, \bar{\alpha}$ and $\bar{\beta}$, can be obtained by minimizing the following equation with respect to $\lambda, \alpha$ and $\beta$

$$
\sum_{i=1}^{n}\left[\ln \left(p_{i}\right)-\ln \left[1-\exp -\left(\frac{\lambda}{e^{\beta x_{i}^{-\alpha}}-1}\right)\right]\right]^{2} .
$$

In percentiles method (PM) of estimate, $p_{i}$ takes a several possible choice as estimates for $F\left(x_{i: n} ; \lambda, \alpha, \beta\right)$, in this study, the formula $p_{i}=\frac{i}{n+1}$, is the expected value of the OGE-IW distribution and will be used.

\section{Numerical Study}

In this section, numerical study is performed to evaluate and compare the performance of the estimates with respect to their biases, and mean square errors (MSEs) for different sample sizes and for different parameter values. The numerical procedures are described through the following algorithm. 
Step(1): A random sample $X_{1}, \ldots, X_{n}$ of sizes $\mathrm{n}=(10,20,30,50,100)$ are selected, these random samples are generated from the OGE-IW distribution by using the following transformation

$x_{i}=\left[\frac{1}{\beta} \ln \left[\frac{-\lambda}{\ln \left(1-u_{i}\right)}+1\right]\right]^{\frac{-1}{\alpha}}, i=1,2, \ldots, n$ and $u_{i}$ are random sample from uniform $(0,1)$.

Step(2): Eight different set values of the parameters are selected as,

$$
\begin{aligned}
& \text { set } 1 \equiv(\lambda=0.2, \alpha=0.5, \beta=0.1), \text { set } 2 \equiv(\lambda=0.2, \alpha=0.5, \beta=0.3), \text { set } 3 \equiv(\lambda=0.2, \alpha=0.5, \beta=0.5), \\
& \text { set } 4 \equiv(\lambda=0.2, \alpha=0.5, \beta=0.7), \text { set } 5 \equiv(\lambda=0.2, \alpha=0.75, \beta=0.3), \text { set } 6 \equiv(\lambda=0.2, \alpha=1, \beta=0.3), \\
& \text { set } 7 \equiv(\lambda=0.2, \alpha=1.25, \beta=0.3) \text { and } \operatorname{set} 8 \equiv(\lambda=0.2, \alpha=1.5, \beta=0.3) .
\end{aligned}
$$

Step(3): For each model parameters and for each sample size, the MLEs, LS estimates and percentiles estimates (PEs) of $\lambda, \alpha$ and $\beta$ are computed.

Step(4): Steps from 1 to 3 are repeated 1000 times for each sample size and for selected sets of parameters. Then, the biases and MSEs of the estimates of the unknown parameters are computed.

Numerical results are reported in Tables (3) to (6) and represented through some Figures from (3) to (6). From these tables, the following conclusions can be observed on the properties of estimated parameters from the OGE-IW distribution.

1- $\quad$ The biases of $\alpha$ in the percentiles method decrease as the value of $\beta$ increases. Also, the biases of $\beta$ increase as the value of $\beta$ increases, for different set of parameters, in approximately all sets of parameters.

2- $\quad$ The biases and MSEs of MLEs, for $\beta$ and $\alpha$ are smaller than the corresponding for $\lambda$.

3- $\quad$ For fixed values of $\lambda, \alpha$ and as the values of $\beta$ increase, the biases and MSEs are decreasing, in approximately most of situations (see Table 4). As the values of $\alpha$ increase and for fixed values ofMSEs for all, the biases and $\beta$ and $\lambda$ estimates decrease in approximately, most sample sizes (see Table 5).

4- The biases and MSEs of ML estimates, for $\beta$ and $\alpha$ are smaller than the corresponding for $\lambda$.

5- The MSEs of the MLEs, LS estimates and PEs decrease as the sample sizes increase for different selected set of parameters (see for example Figures 3 and 4). 


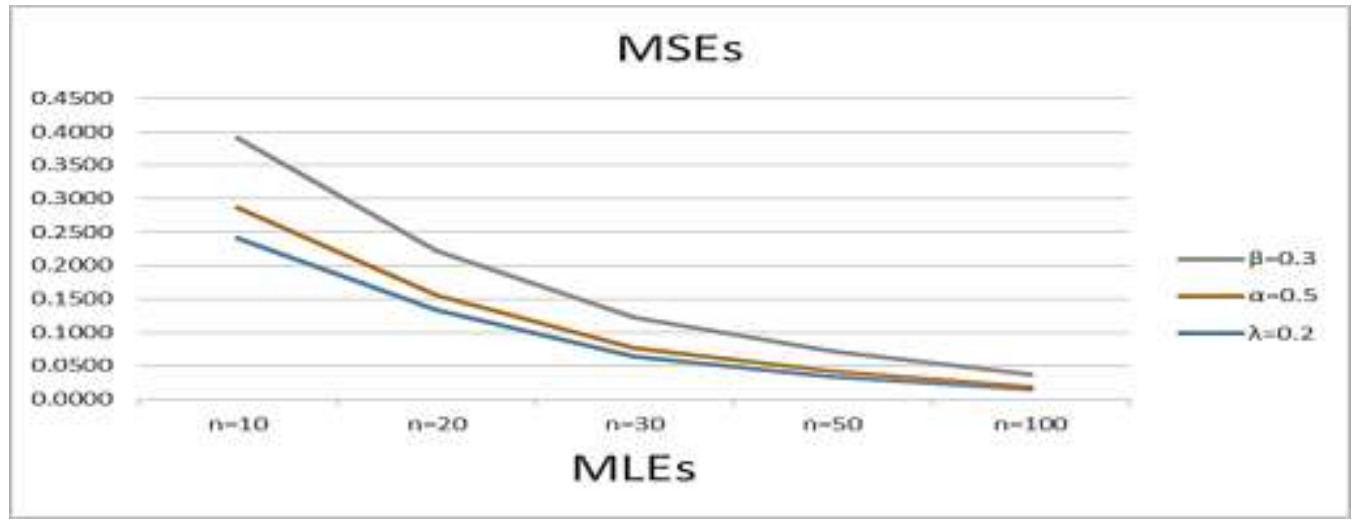

Figure 3: $M S E$ for $M L E$ for the set 2

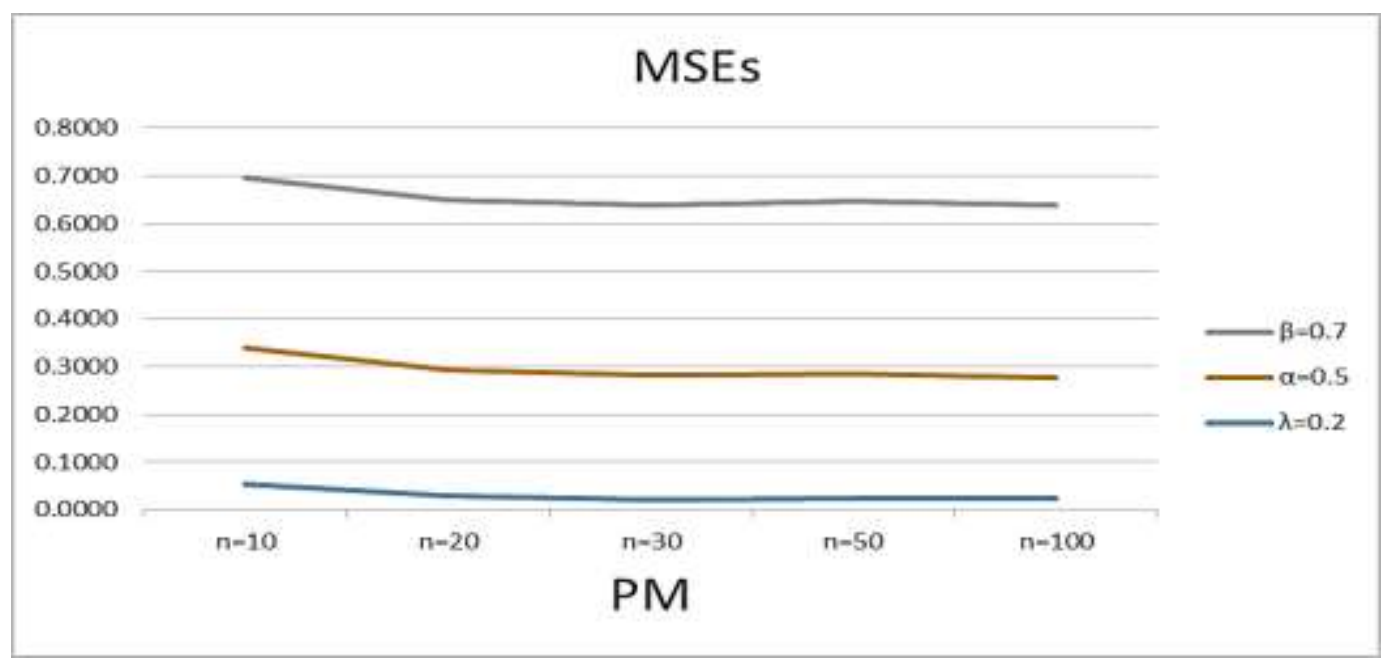

Figure 4: MSE for PE for the set 4

6- The MSEs for the LS estimates, $\beta^{c}$ and $\ell^{\prime}$ take the smallest value among the corresponding MSEs for the other methods in almost all of the cases (see Tables (3) and (4)).

7- $\quad$ The biases of $\alpha$ in the PM decrease as the value of $\beta$ increases. Also, the biases of $\beta$ increase as the value of $\beta$ increases, for different set of parameters, in approximately all sets of parameters.

8- $\quad$ As it seems from Figure (5), the MSEs of the MLEs of $\alpha$ take the smallest values corresponding to the other estimates $\mathscr{E}$ cand $\bar{\alpha}$ for the same sample size and for all set of parameters. Also, from Figure (5) the MSEs of MLEs of $\alpha$ for all set of parameters have the smallest values for the same sample size. Generally, the set 3 of parameters has the smallest MSEs corresponding to other set of parameters. 


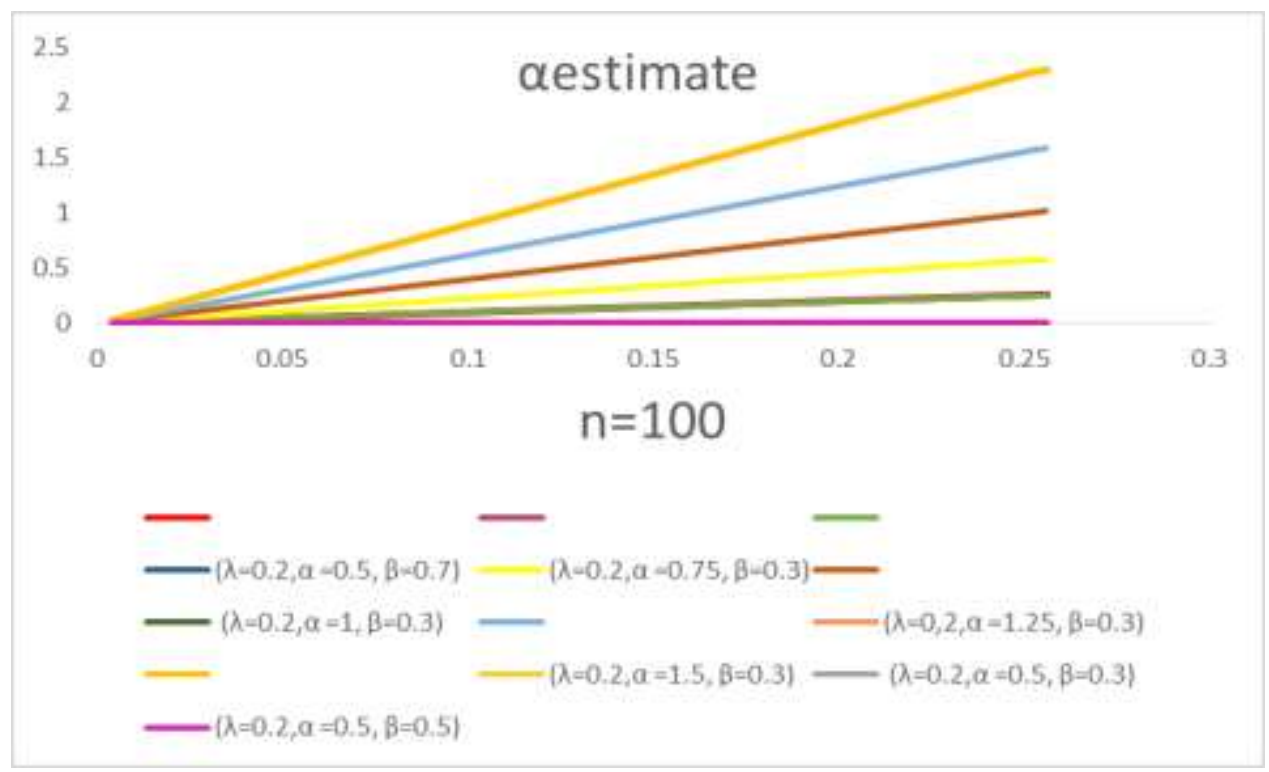

Figure 5: MSEs of $\hat{\alpha}$, Qkand $\bar{\alpha}$ for all set of parameters

9- $\quad$ For fixed values of $\lambda, \alpha$ and as the values of $\beta$ increase, the biases and MSEs are decreasing, in approximately most of situations (see Table 4). As the values of $\alpha$ increase and for fixed values ofMSEs for all, the biases and $\beta$ and $\lambda$ estimates decrease in approximately, most sample sizes (see Table 5).

10- As it seems from Figure (6), the MSEs of the MLEs of $\beta$ take the smallest values corresponding to the other estimates $\beta$ land $\bar{\beta}$ for the same sample size. Also the MSEs of $\beta$ for all sets of parameters have the smallest values for the same sample size. The set 6 of parameters gives the smallest MSEs for different $\beta$ estimates corresponding to other set of parameters.

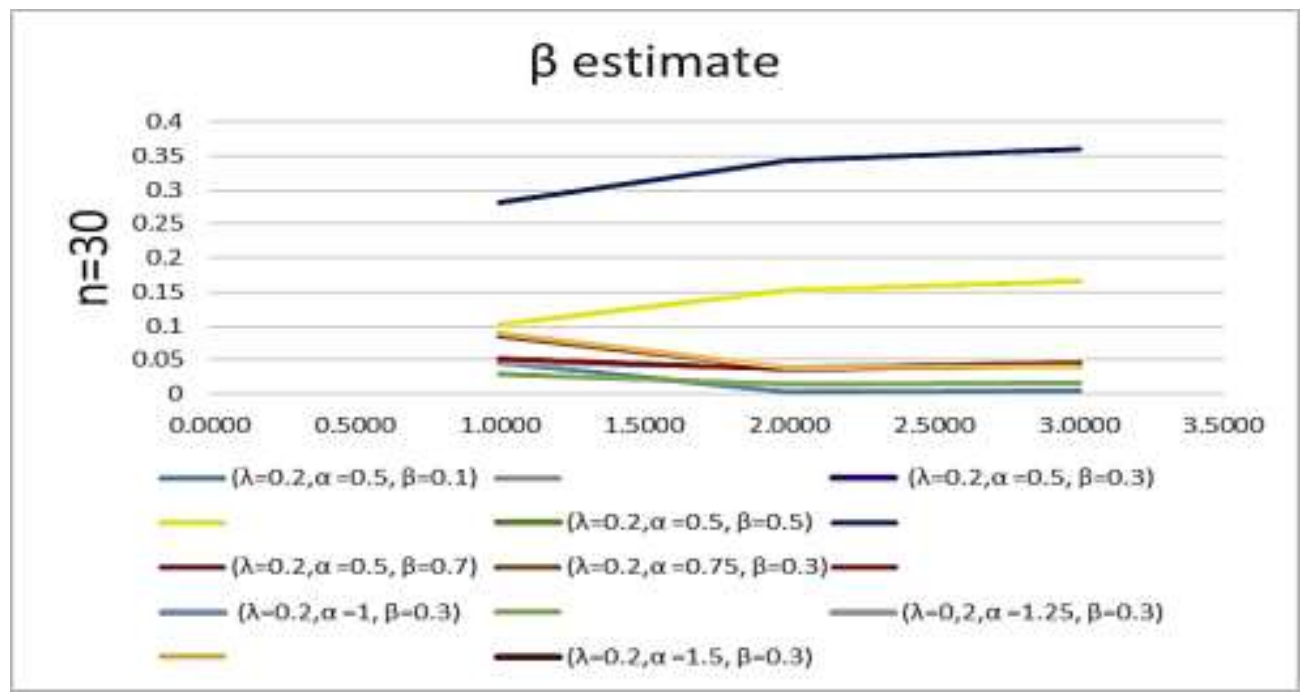

Figure 6: MSEs of $\hat{\beta}, \beta^{\prime}$ and $\bar{\beta}$ for all set of parameters 
Table 3: Biases and MSEs of estimates for set1and set2, for the Odds Generalized Exponential Inverse Weibull distribution

\begin{tabular}{|c|c|c|c|c|c|c|c|c|}
\hline \multirow{2}{*}{\begin{tabular}{|l} 
Sample Size \\
\end{tabular}} & \multirow[b]{2}{*}{ Method } & \multirow[b]{2}{*}{ Properties } & \multicolumn{3}{|c|}{ Set $1 \equiv(\lambda=0.2, \alpha=0.5, \beta=0.1)$} & \multicolumn{3}{|c|}{ Set $2 \equiv(\lambda=0.2, \alpha=0.5, \beta=0.3)$} \\
\hline & & & $\lambda$ & $\alpha$ & $\beta$ & $\lambda$ & $\alpha$ & $\beta$ \\
\hline \multirow{6}{*}{10} & \multirow{2}{*}{ ML } & MSE & 0.8860 & 0.0370 & 0.0770 & 0.2400 & 0.0460 & 0.1050 \\
\hline & & Bias & 0.4290 & 0.0610 & 0.1210 & 0.1120 & 0.0990 & 0.0350 \\
\hline & \multirow{2}{*}{ LS } & MSE & 0.0230 & 0.2850 & 0.0065 & 0.0160 & 0.2690 & 0.0390 \\
\hline & & Bias & -0.1170 & -0.5060 & 0.0070 & -0.1140 & -0.4950 & -0.1850 \\
\hline & \multirow{2}{*}{ PM } & MSE & 0.0530 & 0.3050 & 0.0150 & 0.0310 & 0.2960 & 0.0470 \\
\hline & & Bias & -0.1060 & -0.5110 & 0.0150 & -0.1250 & -0.5030 & -0.1880 \\
\hline \multirow{6}{*}{20} & \multirow{2}{*}{ ML } & MSE & 0.7060 & 0.0200 & 0.0630 & 0.1340 & 0.0210 & 0.0670 \\
\hline & & Bias & 0.3580 & 0.0190 & 0.1110 & 0.0530 & 0.0650 & -0.0017 \\
\hline & \multirow{2}{*}{ LS } & MSE & 0.0170 & 0.2560 & 0.0041 & 0.0160 & 0.2560 & 0.0390 \\
\hline & & Bias & -0.1200 & -0.4970 & 0.0046 & -0.1140 & -0.4960 & -0.1850 \\
\hline & \multirow{2}{*}{ PM } & MSE & 0.0290 & 0.2670 & 0.0082 & 0.0270 & 0.2650 & 0.0480 \\
\hline & & Bias & -0.1330 & -0.5000 & 0.0046 & -0.1360 & -0.4980 & -0.1920 \\
\hline \multirow{6}{*}{30} & \multirow{2}{*}{ ML } & MSE & 0.5010 & 0.0140 & 0.0470 & 0.0640 & 0.0130 & 0.0460 \\
\hline & & Bias & 0.2660 & 0.0110 & 0.0850 & 0.0018 & 0.0540 & -0.0380 \\
\hline & \multirow{2}{*}{$\mathrm{LS}$} & MSE & 0.0180 & 0.2530 & 0.0039 & 0.0150 & 0.2560 & 0.0380 \\
\hline & & Bias & -0.1220 & -0.4960 & 0.0023 & -0.1130 & -0.4960 & -0.1840 \\
\hline & \multirow{2}{*}{$\mathrm{PM}$} & MSE & 0.0250 & 0.2610 & 0.0056 & 0.0240 & 0.2600 & 0.0460 \\
\hline & & Bias & -0.1440 & -0.4990 & -0.0021 & -0.1460 & -0.4980 & -0.2000 \\
\hline \multirow{6}{*}{50} & \multirow{2}{*}{ ML } & MSE & 0.4320 & 0.0086 & 0.0320 & 0.0340 & 0.0074 & 0.0310 \\
\hline & & Bias & 0.1850 & 0.0047 & 0.0600 & -0.0300 & 0.0430 & -0.0620 \\
\hline & \multirow{2}{*}{$\mathrm{LS}$} & MSE & 0.0180 & 0.2570 & 0.0042 & 0.0150 & 0.2560 & 0.0370 \\
\hline & & Bias & -0.1210 & -0.5030 & 0.0050 & -0.1100 & -0.5030 & -0.1800 \\
\hline & \multirow{2}{*}{ PM } & MSE & 0.0240 & 0.2620 & 0.0042 & 0.0240 & 0.2620 & 0.0450 \\
\hline & & Bias & -0.1490 & -0.5060 & -0.0045 & -0.1500 & -0.5050 & -0.2030 \\
\hline \multirow{6}{*}{100} & \multirow{2}{*}{ ML } & MSE & 0.1420 & 0.0044 & 0.0160 & 0.0150 & 0.0036 & 0.0190 \\
\hline & & Bias & 0.1040 & -0.0012 & 0.0370 & -0.0540 & 0.0350 & -0.0780 \\
\hline & \multirow{2}{*}{ LS } & MSE & 0.0170 & 0.2530 & 0.0043 & 0.0130 & 0.2530 & 0.0330 \\
\hline & & Bias & -0.1200 & -0.5020 & 0.0079 & -0.1010 & -0.5010 & -0.1690 \\
\hline & \multirow{2}{*}{ PM } & MSE & 0.0240 & 0.2560 & 0.0039 & 0.0230 & 0.2560 & 0.0430 \\
\hline & & Bias & -0.1500 & -0.5020 & -0.0015 & -0.1490 & -0.5020 & -0.2000 \\
\hline
\end{tabular}


Table 4: Biases and MSEs of estimates for set3and set4, for the Odds Generalized Exponential Inverse Weibull distribution

\begin{tabular}{|c|c|c|c|c|c|c|c|c|}
\hline & & & Set $3 \equiv(\lambda$ & $=0.2, \alpha=0$. & $, \beta=0.5)$ & Set $4 \equiv(\lambda$ & $=0.2, \alpha=0$. & $\beta=0.7)$ \\
\hline Sample Size & Method & Properties & $\lambda$ & $\alpha$ & $\beta$ & $\lambda$ & $\alpha$ & $\beta$ \\
\hline \multirow{6}{*}{10} & \multirow{2}{*}{ ML } & MSE & 0.0650 & 0.0540 & 0.1300 & 0.0460 & 0.0560 & 0.2800 \\
\hline & & Bias & -0.0780 & 0.1370 & -0.2410 & -0.1370 & 0.1480 & -0.4850 \\
\hline & \multirow{2}{*}{ LS } & MSE & 0.0200 & 0.2710 & 0.1500 & 0.0170 & 0.2660 & 0.3420 \\
\hline & & Bias & -0.1050 & -0.4950 & -0.3760 & -0.1060 & -0.4930 & -0.5800 \\
\hline & \multirow{2}{*}{ PM } & MSE & 0.0750 & 0.2890 & 0.1600 & 0.0550 & 0.2840 & 0.3570 \\
\hline & & Bias & -0.1180 & -0.4990 & -0.3870 & -0.1110 & -0.4950 & -0.5830 \\
\hline \multirow{6}{*}{20} & \multirow{2}{*}{ ML } & MSE & 0.0360 & 0.0250 & 0.1080 & 0.0290 & 0.0260 & 0.2730 \\
\hline & & Bias & -0.1000 & 0.0980 & -0.2570 & -0.1510 & 0.1090 & -0.5010 \\
\hline & \multirow{2}{*}{ LS } & MSE & 0.0170 & 0.2550 & 0.1500 & 0.0170 & 0.2550 & 0.3420 \\
\hline & & Bias & -0.1080 & -0.4950 & -0.3790 & -0.1090 & -0.4950 & -0.5800 \\
\hline & \multirow{2}{*}{ PM } & MSE & 0.0250 & 0.2640 & 0.1630 & 0.0300 & 0.2620 & 0.3570 \\
\hline & & Bias & -0.1380 & -0.4960 & -0.3940 & -0.1290 & -0.4950 & -0.5850 \\
\hline \multirow{6}{*}{30} & \multirow{2}{*}{ ML } & MSE & 0.0260 & 0.0160 & 0.1020 & 0.0270 & 0.0170 & 0.2810 \\
\hline & & Bias & -0.1110 & 0.0820 & -0.2660 & -0.1590 & 0.0940 & -0.5200 \\
\hline & \multirow{2}{*}{ LS } & MSE & 0.0500 & 0.2520 & 0.1530 & 0.0220 & 0.2520 & 0.3430 \\
\hline & & Bias & -0.1040 & -0.4950 & -0.3780 & -0.1070 & -0.4950 & -0.5790 \\
\hline & \multirow{2}{*}{ PM } & MSE & 0.0230 & 0.2590 & 0.1650 & 0.0220 & 0.2590 & 0.3590 \\
\hline & & Bias & -0.1450 & -0.4970 & -0.3990 & -0.1420 & -0.4970 & -0.5950 \\
\hline \multirow{6}{*}{50} & \multirow{2}{*}{ ML } & MSE & 0.0200 & 0.0096 & 0.0940 & 0.0270 & 0.0110 & 0.2830 \\
\hline & & Bias & -0.1200 & 0.0690 & -0.2760 & -0.1610 & 0.0800 & -0.5220 \\
\hline & \multirow{2}{*}{ LS } & MSE & 0.0150 & 0.2560 & 0.1480 & 0.0160 & 0.2560 & 0.3400 \\
\hline & & Bias & -0.1080 & -0.5030 & -0.3780 & -0.1070 & -0.5030 & -0.5780 \\
\hline & \multirow{2}{*}{ PM } & MSE & 0.0230 & 0.2610 & 0.1660 & 0.0230 & 0.2610 & 0.3640 \\
\hline & & Bias & -0.1500 & -0.5050 & -0.4040 & -0.1470 & -0.5040 & -0.6000 \\
\hline \multirow{6}{*}{100} & \multirow{2}{*}{ ML } & MSE & 0.0190 & 0.0056 & 0.0920 & 0.0270 & 0.0069 & 0.2910 \\
\hline & & Bias & -0.1250 & 0.0590 & -0.2810 & -0.1640 & 0.0700 & -0.5350 \\
\hline & \multirow{2}{*}{ LS } & MSE & 0.0170 & 0.2530 & 0.1470 & 0.0150 & 0.2530 & 0.3370 \\
\hline & & Bias & -0.1040 & -0.5010 & -0.3750 & -0.1050 & -0.5010 & -0.5750 \\
\hline & \multirow{2}{*}{ PM } & MSE & 0.0230 & 0.2550 & 0.1650 & 0.0230 & 0.2550 & 0.3620 \\
\hline & & Bias & -0.1500 & -0.5020 & -0.4020 & -0.1480 & -0.5020 & -0.5990 \\
\hline
\end{tabular}


Table 5: Biases and MSEs of estimates for set5and set6, for the Odds Generalized Exponential Inverse Weibull distribution

\begin{tabular}{|c|c|c|c|c|c|c|c|c|}
\hline & & & Set $5 \equiv($ & $0.2, \alpha=0$. & $\beta=0.3)$ & Set6 & $=0.2, \alpha=1$ & $=0.3)$ \\
\hline Sample Size & Method & Properties & $\lambda$ & $\alpha$ & $\beta$ & $\lambda$ & $\alpha$ & $\beta$ \\
\hline \multirow{6}{*}{10} & \multirow{2}{*}{ ML } & MSE & 0.3540 & 0.1070 & 0.1370 & 0.3190 & 0.1860 & 0.1040 \\
\hline & & Bias & 0.1810 & 0.1390 & 0.0740 & 0.1050 & 0.2060 & 0.0110 \\
\hline & \multirow{2}{*}{ LS } & MSE & 0.0150 & 0.6060 & 0.0370 & 0.0150 & 1.0770 & 0.0370 \\
\hline & & Bias & -0.1100 & -0.7430 & -0.1800 & -0.1090 & -0.9900 & -0.1780 \\
\hline & \multirow{2}{*}{ PM } & MSE & 0.0390 & 0.6570 & 0.0480 & 0.0320 & 1.1690 & 0.0450 \\
\hline & & Bias & -0.1130 & -0.7530 & -0.1750 & -0.1140 & -1.0020 & -0.1720 \\
\hline \multirow{6}{*}{20} & \multirow{2}{*}{ ML } & MSE & 0.2520 & 0.0480 & 0.1110 & 0.1710 & 0.0830 & 0.0670 \\
\hline & & Bias & 0.1370 & 0.0750 & 0.0570 & 0.0420 & 0.1300 & -0.0230 \\
\hline & \multirow{2}{*}{ LS } & MSE & 0.0150 & 0.5780 & 0.0380 & 0.0150 & 1.0270 & 0.0380 \\
\hline & & Bias & -0.1120 & -0.7450 & -0.1830 & -0.1110 & -0.9930 & -0.1820 \\
\hline & \multirow{2}{*}{ PM } & MSE & 0.0260 & 0.2700 & 0.0470 & 0.0290 & 1.0640 & 0.0460 \\
\hline & & Bias & -0.1340 & 0.6010 & 0.0470 & -0.1330 & -0.9960 & -0.1890 \\
\hline \multirow{6}{*}{30} & \multirow{2}{*}{ ML } & MSE & 0.1690 & 0.0300 & 0.0860 & 0.0890 & 0.0500 & 0.0510 \\
\hline & & Bias & 0.0990 & 0.0520 & 0.0400 & 0.0073 & 0.0990 & -0.0370 \\
\hline & \multirow{2}{*}{ LS } & MSE & 0.0150 & 0.5790 & 0.0370 & 0.0150 & 1.0290 & 0.0370 \\
\hline & & Bias & -0.1090 & -0.7520 & -0.1790 & -0.1090 & -1.0030 & -0.1780 \\
\hline & \multirow{2}{*}{ PM } & MSE & 0.0270 & 0.5970 & 0.0460 & 0.0230 & 1.0620 & 0.0430 \\
\hline & & Bias & -0.1390 & -0.7550 & -0.1920 & -0.1380 & -1.0070 & -0.1870 \\
\hline \multirow{6}{*}{50} & \multirow{2}{*}{ ML } & MSE & 0.1020 & 0.0200 & 0.0590 & 0.0330 & 0.0310 & 0.0280 \\
\hline & & Bias & 0.0470 & 0.0430 & 0.0031 & -0.0410 & 0.0880 & -0.0720 \\
\hline & \multirow{2}{*}{$\mathrm{LS}$} & MSE & 0.0150 & 0.5770 & 0.0380 & 0.0150 & 1.0260 & 0.0370 \\
\hline & & Bias & -0.1130 & -0.7550 & -0.1840 & -0.1110 & -1.0060 & -0.1810 \\
\hline & \multirow{2}{*}{ PM } & MSE & 0.0230 & 0.5870 & 0.0430 & 0.0220 & 1.0440 & 0.0420 \\
\hline & & Bias & -0.1480 & -0.7570 & -0.2000 & -0.1450 & -1.0090 & -0.1950 \\
\hline \multirow{6}{*}{100} & \multirow{2}{*}{ ML } & MSE & 0.0400 & 0.0091 & 0.0320 & 0.0150 & 0.0150 & 0.0180 \\
\hline & & Bias & 0.0052 & 0.0320 & -0.0200 & 0.0410 & 0.0700 & -0.0820 \\
\hline & \multirow{2}{*}{ LS } & MSE & 0.0150 & 0.5680 & 0.0370 & 0.0140 & 1.0100 & 0.0360 \\
\hline & & Bias & -0.1110 & -0.7510 & -0.1810 & -0.0820 & -0.0820 & -0.1770 \\
\hline & \multirow{2}{*}{ PM } & MSE & 0.0230 & 0.5720 & 0.0430 & 0.0220 & 1.0170 & 0.0410 \\
\hline & & Bias & -0.1500 & -0.7510 & -0.2000 & -0.1450 & -1.0020 & -0.1920 \\
\hline
\end{tabular}


Table 6: Biases and MSEs of estimates for set7and set8, for the Odds Generalized Exponential Inverse Weibull distribution

\begin{tabular}{|c|c|c|c|c|c|c|c|c|}
\hline & & & Set7 $\equiv($ & $0.2, \alpha=1.2$ & $\beta=0.3)$ & Set $8 \equiv$ & $0.2, \alpha=1$ & $=0.3)$ \\
\hline Sample Size & Method & Properties & $\lambda$ & $\alpha$ & $\beta$ & $\lambda$ & $\alpha$ & $\beta$ \\
\hline \multirow{6}{*}{10} & \multirow{2}{*}{ ML } & MSE & 0.1240 & 0.2790 & 0.2790 & 0.5260 & 0.4040 & 0.1700 \\
\hline & & Bias & -0.0089 & 0.2890 & -0.0500 & 0.2100 & 0.2730 & 0.0930 \\
\hline & \multirow{2}{*}{ LS } & MSE & 0.9730 & 1.4260 & 1.4260 & 0.0160 & 2.4260 & 0.0380 \\
\hline & & Bias & -0.0500 & -1.1360 & -0.1360 & -0.1100 & -1.4860 & -0.1800 \\
\hline & \multirow{2}{*}{ PM } & MSE & 1.4260 & 1.3760 & 0.0230 & 0.0370 & 2.5950 & 0.0460 \\
\hline & & Bias & 0.5750 & -1.0910 & -0.1350 & -0.1080 & -1.5000 & -0.1650 \\
\hline \multirow{6}{*}{20} & \multirow{2}{*}{ ML } & MSE & 0.0250 & 0.0250 & 0.0290 & 0.3260 & 0.1870 & 0.1150 \\
\hline & & Bias & -0.0550 & 0.1910 & -0.0790 & 0.1280 & 0.1640 & 0.0420 \\
\hline & \multirow{2}{*}{ LS } & MSE & 0.3690 & 1.5120 & 0.0170 & 0.0160 & 2.3140 & 0.0390 \\
\hline & & Bias & 0.1010 & -1.2010 & -0.1230 & -0.1130 & -1.4910 & -0.1840 \\
\hline & \multirow{2}{*}{ PM } & MSE & 0.7390 & 1.4770 & 0.0200 & 0.0290 & 2.3920 & 0.0460 \\
\hline & & Bias & 0.2380 & -1.1670 & -0.1230 & -0.1270 & -1.4940 & -0.1810 \\
\hline \multirow{6}{*}{30} & \multirow{2}{*}{ ML } & MSE & 0.0330 & 0.0730 & 0.0300 & 0.2020 & 0.1160 & 0.0900 \\
\hline & & Bias & -0.0490 & 0.1440 & -0.0740 & 0.0790 & 0.1230 & 0.0200 \\
\hline & \multirow{2}{*}{ LS } & MSE & 0.1360 & 1.5800 & 0.0150 & 0.0160 & 2.3180 & 0.0390 \\
\hline & & Bias & -0.0001 & -1.2410 & -0.1180 & -0.1130 & -1.5050 & -0.1840 \\
\hline & \multirow{2}{*}{ PM } & MSE & 0.3430 & 1.5750 & 0.0160 & 0.0240 & 2.3850 & 0.0410 \\
\hline & & Bias & 0.0530 & -1.2230 & -0.1200 & -0.1340 & -1.5090 & -0.1820 \\
\hline \multirow{6}{*}{50} & \multirow{2}{*}{ ML } & MSE & 0.0180 & 0.0460 & 0.0210 & 0.0720 & 0.0710 & 0.0410 \\
\hline & & Bias & -0.0620 & 0.1190 & -0.0870 & 0.0000 & 0.1120 & -0.0360 \\
\hline & \multirow{2}{*}{ LS } & MSE & 0.0140 & 1.5970 & 0.0140 & 0.0160 & 2.3090 & 0.0400 \\
\hline & & Bias & -0.0550 & -1.2560 & -0.1160 & -0.1150 & -1.5100 & -0.1870 \\
\hline & \multirow{2}{*}{ PM } & MSE & 0.0800 & 1.6100 & 0.0150 & 0.0220 & 2.3490 & 0.0410 \\
\hline & & Bias & -0.0720 & -1.2530 & -0.1180 & -0.1420 & -1.5130 & -0.1900 \\
\hline \multirow{6}{*}{100} & \multirow{2}{*}{ ML } & MSE & 0.0078 & 0.0210 & 0.0120 & 0.0280 & 0.0310 & 0.0210 \\
\hline & & Bias & -0.0650 & 0.0890 & -0.0860 & -0.0320 & 0.0860 & -0.0540 \\
\hline & \multirow{2}{*}{ LS } & MSE & 0.0035 & 1.5760 & 0.0130 & 0.0170 & 2.2740 & 0.0420 \\
\hline & & Bias & -0.0580 & -1.2520 & -0.1130 & -0.1200 & -1.5030 & -0.1930 \\
\hline & \multirow{2}{*}{ PM } & MSE & 0.0110 & 1.5890 & 0.0140 & 0.0220 & 2.2890 & 0.0400 \\
\hline & & Bias & -0.1060 & -1.2530 & -0.1200 & -0.1430 & -1.5030 & -0.1890 \\
\hline
\end{tabular}




\section{Data Analysis}

In this section, we provide a data analysis in order to assess the goodness-of-fit of the OGE-IW model comparing with some known distributions such as the exponential (E) generalized exponential (GE) generalized inverse Weibull (GIW), Kumaraswamy inverse Weibull (KIW), Marshpall-Olkin extended inverse Weibull (MOEIW) and IW. The data set refers to Lee and Wang (2003) which represent remission times (in months) of a random sample of 128 bladder cancer patients. The data are as follows:

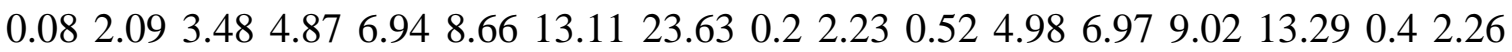
3.575 .067 .090 .2213 .825 .740 .52 .463 .465 .097 .269 .4714 .240 .820 .512 .543 .75 .17

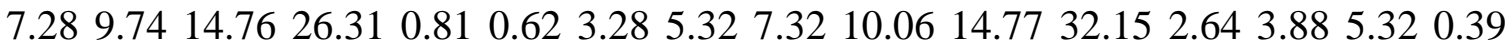

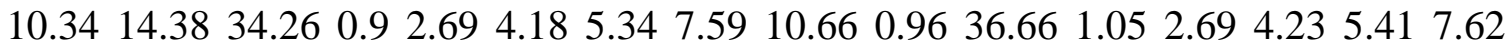
$10.7516 .6243 .010 .192 .754 .265 .417 .63 \quad 17.1246 .121 .262 .834 .33 \quad 0.6611 .2517 .14$ 79.051 .352 .875 .627 .8711 .6417 .360 .43 .024 .345 .717 .9311 .7918 .11 .464 .45 .85 0.2611 .9819 .131 .763 .254 .56 .258 .3712 .022 .020 .314 .516 .548 .5312 .0320 .282 .02 3.366 .7612 .070 .732 .073 .366 .398 .6512 .6322 .695 .49 .

Measures of fit statistic using the maximized $\log$-likelihood $(-2 \log L)$, Akaike information criterion $(A I C)$, the corrected Akaike information criterion $(C A I C)$, and Hannan-Quinn information criterion (HQIC), are provided in Table 7. The model with minimum values for $-2 \log L$ or $A I C$ or $B I C$ or $C A I C$ or $H Q I C$ can be chosen as the best model to fit the data. The ML estimates and their standard errors (SE) for OGE-IW, GE, E, GIW, KIW, MOEIW and IW models are given in Table 8.

Table 7: The statistics-2log $L, A I C, C A I C, B I C$, and $H Q I C$, for the 128 bladder cancer patients data

\begin{tabular}{|c|c|c|c|c|c|}
\hline Distribution & $-2 \log L$ & $A I C$ & $B I C$ & $C A I C$ & $H Q I C$ \\
\hline OGE-IW & 801.263 & 807.263 & 807.585 & 807.457 & 810.740 \\
\hline GE & 805.022 & 809.022 & 809.236 & 809.118 & 811.339 \\
\hline E & 827.296 & 829.296 & 829.403 & 829.328 & 830.455 \\
\hline GIW & 874.450 & 880.450 & 863.673 & 880.644 & 883.926 \\
\hline KIW & 971.574 & 979.574 & 980.003 & 979.899 & 984.209 \\
\hline MOEIW & 810.707 & 816.707 & 817.029 & 816.901 & 820.183 \\
\hline IW & 857.352 & 861.352 & 861.566 & 861.448 & 863.669 \\
\hline
\end{tabular}


Table 8: ML estimates of the model parameters and the corresponding SEs for the 128 bladder cancer patient's data

\begin{tabular}{|c|c|c|c|c|c|c|}
\hline Distribution & $\hat{\lambda}$ & $\hat{\alpha}$ & $\hat{\beta}$ & $\hat{a}$ & $\hat{b}$ & $\hat{\theta}$ \\
\hline OGE-IW & 0.057 & 0.87 & 0.336 & - & - & - \\
& $(0.05)$ & $(0.076)$ & $(0.244)$ & - & - & - \\
\hline GE & 0.111 & 0.922 & - & - & - & - \\
& $(0.013)$ & $(0.107)$ & - & - & - & - \\
\hline E & 0.075 & - & - & - & - & - \\
& $(0.008)$ & - & - & - & - & - \\
\hline GIW & 0.75 & 0.53 & 1.797 & - & - & - \\
& $(0.25)$ & $(0.038)$ & $(0.324)$ & - & - & - \\
\hline KIW & - & 3.796 & 2.239 & 0.0077 & 0.093 & - \\
& - & $(0.238)$ & $(2.846)$ & $(0.006)$ & $(0.012)$ & - \\
\hline MOEIW & - & 0.047 & 1.39 & - & - & 198.304 \\
& - & $(0.049)$ & $(0.104)$ & - & - & $(221.399)$ \\
\hline IW & 16.142 & 0.464 & - & - & - & - \\
& $(0.125)$ & $(0.042)$ & - & - & - & - \\
\hline
\end{tabular}

The results show that the OGE-IW distribution provides a significantly better fit than the other models.

\section{Conclusion}

In this article, we propose a new model, called the odds generalized exponential-inverse Weibull distribution based on T-X family presented by Alzaatreh et al. (2013). Some statistical properties of current distribution are derived and discussed. The estimation of the model parameters is approached by maximum likelihood, least squares and percentiles methods. Simulation study is carried out to compare the performance of different estimates. Simulation study revealed that the PEs perform well than the MLEs and LS estimates, in approximately, most of situations. An application to a real data set indicates that the new model is superior to the fits than the other well-known distributions.

\section{References}

1. Alizadeh, M., Ghosh, I., Yousof, H. M., Rasekhi, M., and Hamedani, G. G. (2017). The generalized odd generalized exponential family of distributions: Properties, characterizations and application. Journal of Data Science, 15(3), 443-465.

2. Alzaatreh, A., Lee, C. and Famoye, F. (2013). A new method for generating families of continuous distributions. Metron, 71, 63-79. 
3. Calabria, R., and Pulcini, G. (1990). On the maximum likelihood and least squares estimation in the inverse Weibull distribution, Statistica Applicata, 2, 5366.

4. Calabria, R., and Pulcini, G. (1994). Bayes two-sample prediction for the inverse Weibull distribution. Communications in Statistics-Theory \& Methods, 23, 1811-1824.

5. de Gusmão, F. R. S., Ortega, E. M. M., and Cordeiro, G. M. (2011). The generalized inverse Weibull distribution. Statistical Papers, 52, 591-619.

6. Elbatal, I., and Muhammed, H. Z. (2014). Exponentiated generalized inverse Weibull distribution. Applied Mathematical Sciences, 8, 3997-4012.

7. Greenwood, J. A., Landwehr, J. M. and Matalas, N. C. (1979) probability weighted moments: Definitions and relations of parameters of several distributions expressible in inverse form. Water Resources Research, 15, 10491054.

8. Gupta, R. D., and Kundu, D. (1999). Generalized exponential distribution. Australian and New Zealand Journal of Statistics, 41 (2), 173-188.

9. Gupta, R. C, Gupta, P. I. and Gupta, R. D. (1998). Modeling failure time data by Lehmann alternatives. Communications in Statistics-Theory and Methods, 27, 887-904.

10. Hassan, A. S., and Al-Thobety, A. K. (2012). Optimal design of failure step stress partially accelerated life tests with type II inverted Weibull data. International Journal of Engineering Research and Applications, 2(3), 3242-3253.

11. Hassan, A. S., Assar, M. S., and Zaky, A. N. (2015)) constant-stress partially accelerated life tests with type II inverted Weibull distribution with multiple censored data. International Journal of Advanced Statistics and Probability, 3(1), 72-82.

12. Keller, A. Z., and Kamath, A. R. (1982). Reliability analysis of CNC machine tools. Reliability engineering, 3, 449-473.

13. Khan, M. S. (2010). The beta inverse Weibull distribution. International Transactions in Mathematical Sciences and Computer, 3,113-119.

14. Khan, M. S., and King, R. (2012). Modified inverse Weibull distribution. Journal of Statistics Applications \& Probability, 1, 115-132.

15. Khan, M. S., and King, R. (2016). New generalized inverse Weibull distribution for lifetime modeling. Communications for Statistical Applications and Methods, 23(2), 147-161

16. Khan, M. S., King, R., and Hudson, I. L. (2014). Characterizations of the transmuted inverse Weibull distribution, ANZIAM Journal, 55, 197-217.

17. Lee, E. T., and Wang J. W. (2003). Statistical Methods for Survival Data Analysis. 3rd edition, Wiley, New York, http://dx.doi.org/10.1002/0471458546.

18. Maiti, S. S., and Pramanik, S. (2015). Odds generalized exponential-exponential distribution. Journal of Data Science, 13, 733-279. 
19. Marshall, A.W and Olkin, I. (1997). A new method for adding a parameter to a family of distributions with applications to the exponential and Weibull families. Biometrika, 84, 641-652.

20. Okasha, H. M., El-Baz, A.H., Tarabia, A. M. K., Basheer, A. M. (2017). Extended inverse Weibull distribution with reliability application. Journal of the Egyptian Mathematical Society, 25, 343-349.

21. Oluyede, B. O., and Yang, T. (2014). Generalizations of the inverse Weibull and related distributions with applications. Electronic Journal of Applied Statistical Analysis, 7(1), 94-116.

22. Pararai, M., Warahena-Liyanage, G., and Oluyede, B.O. (2014). A new class of generalized inverse Weibull distribution with applications. Journal of Applied Mathematics \& Bioinformatics, 4(2), 17-35.

23. Rodrigues, J. A., Silva, A. P. C. M., and Hamedani, G. G. (2016). The exponentiated Kumaraswamy inverse Weibull distribution with application in survival analysis. Journal of Statistical Theory and Applications, 15(1), 8-24.

24. Shahbaz, M. Q., Shahbaz, S. and Butt, N. S. (2012). The Kumaraswamyinverse Weibull distribution. Pakistan Journal of Statistics and Operation Research, 8(3), 479-489.

25. Tahir, M. H., Cordeiro, G. M., Alizadeh, M., Mansoor, M., Zubair, M. and Hamedani, G. G. (2015).The odd generalized exponential family of distributions with applications. Journal of Statistical Distributions and Applications, 2(1), 128. 